\title{
Estructura Factorial, Invarianza y Propiedades Psicométricas de la Escala de Autoestima de Rosenberg en el Contexto Ecuatoriano
}

\author{
Factor Structure, Invariance and Psychometric Properties of the Rosenberg Self- \\ Esteem Scale in the Ecuadorian Context
}

\author{
Alexandra Bueno-Pacheco ${ }^{1}$, Sandra Lima-Castro ${ }^{2}$, Paúl Arias-Medina ${ }^{3}$, Eva Peña-Contreras ${ }^{4}$, Mónica \\ Aguilar-Sizer ${ }^{5}$ y Marcela Cabrera-Vélez ${ }^{6}$
}

\begin{abstract}
Resumen
El objetivo del presente estudio fue evaluar las propiedades psicométricas de la Escala de Autoestima de Rosenberg (EAR; Rosenberg, 1965) en el contexto ecuatoriano. Para ello, se evaluó la versión original junto con modelos alternativos propuestos por otras investigaciones en una muestra de 535 adultos en la ciudad de Cuenca, Ecuador. Los resultados del análisis factorial confirmatorio muestran que el modelo bifactor presenta buena consistencia interna y adecuada bondad de ajuste. La validez de criterio se evaluó correlacionando las dimensiones de autoestima positiva y autoestima negativa con las subescalas de dominio psicológico de Calidad de Vida WHOQOL-BREF, Apoyo Social de DUKE y Compromiso con la Vida. Se concluye que la autoestima medida con la EAR es esencialmente unidimensional y que posee la propiedad de invarianza para la variable sexo.
\end{abstract}

Palabras clave: EAR, autoestima, autoconfianza, autodesprecio, propiedades psicométricas

\begin{abstract}
The aim of the present study was to evaluate the psychometric properties of the Rosenberg Self-Esteem Scale (RSE, Rosenberg, 1965) in the Ecuadorian context. The original version was evaluated along with alternative models proposed by other research in a sample of 535 adults in the city of Cuenca, Ecuador. The results of the confirmatory factor analysis show that the bifactor model has good internal consistency and adequate goodness of fit. Criterion validity was evaluated by correlating the dimensions of positive selfesteem and negative self-esteem with the subscales of the psychological domain of Quality of Life WHOQOL-BREF, Social Support of DUKE and Engagement to Life. Self-esteem measured by the RSE is essentially unidimensional and is invariant between the sex categories.
\end{abstract}

Keywords: RSE, self-esteem, self-confidence, self-deprecation, psychometric properties

\footnotetext{
${ }^{1}$ Magíster en Diagnóstico y Rehabilitación Neuropsicológica. Doctoranda en Psicogerontología. Docente-Investigadora de la Universidad del Azuay, Facultad de Filosofía, Letras y Ciencias de la Educación. Av. 24 de mayo 7-77 y Hernán Malo. Apartado: 01.01.981. Ecuador. Tel.: +593991506 998. Correo: abueno@ uazuay.edu.ec (correpondencia)

${ }^{2}$ Magíster en Psicoterapia Integrativa y Psicología de la Salud, Doctoranda en Neurociencias y Psicología Clínica. Docente-Investigadora de la Universidad de Cuenca. Ecuador. Correo: sandra.lima@ucuenca.edu.ec (correpondencia)

${ }^{3}$ Máster en Estudios del Desarrollo. Docente Investigador de la Universidad de Cuenca. Ecuador.

${ }^{4}$ Magíster en psicología de la educación y el desarrollo humano en contextos multiculturales. Docente-Investigadora de la Universidad de Cuenca. Ecuador.

${ }^{5}$ Magíster en Intervención, Asesoría y Terapia Familiar Sistémica. Docente Titular de la Universidad de Cuenca y Directora de la Carrera de Psicología Clínica. Ecuador.

${ }^{6}$ Magíster en Psicoterapia Integrativa. Doctoranda en Psicología Clínica y de la Salud. Docente-Investigadora, Universidad de Cuenca. Ecuador.
}

Revista Iberoamericana de Diagnóstico y Evaluación - e Avaliação Psicológica. RIDEP · №56 • Vol.3 · 87-100 2020

ISSN: 1135-3848 print /2183-6051online 


\section{Introducción}

El estudio de la autoestima es esencial en la investigación en tanto ha sido relacionada con diversas áreas de interés; por ejemplo se ha señalado que está asociada con una buena salud mental (Keane \& Loades, 2017), con la mejoría en la salud de pacientes con enfermedades crónicas (Robinson, et al., 2019), e incluso puede contribuir al alcance de mayores ingresos económicos al aumentar el esfuerzo y la productividad de las personas (Drago, 2011; Waddell, 2006).

La autoestima es un constructo que implica sentimientos de estima propia, con una autoevaluación basada en aspectos positivos y negativos de uno mismo (Baumeister et al., 1998; Rosenberg, 1965). Individuos con alta autoestima se respetan a sí mismos, reconocen sus propias limitaciones y tienen expectativas de mejora y crecimiento personal (Rosenberg et al., 1995).

Varias investigaciones señalan la trascendencia de la autoestima en el bienestar general, se ha señalado que las personas con alta autoestima tienen más probabilidades de estar felices y manifestar bienestar psicológico (BrajsaZganec et al., 2017; Oh et al., 2017). Una alta autoestima tiene un efecto positivo en las relaciones de tipo romántico al influir en la felicidad de la pareja (Erol \& Orth, 2016). Además, puede ser considerada como un factor protector frente a trastornos mentales como la ansiedad, depresión, y una amplia gama de síntomas como falta de motivación, intentos de suicidio, etc. (Keane \& Loades, 2017; Palacios et al., 2018; Pereira et al., 2018).

Por otro lado, algunos investigadores indican que la baja autoestima es un predictor de depresión y ansiedad (Sowislo \& Orth, 2012). Además, la baja autoestima en la adolescencia es un predictor de comportamiento delictivo, mala salud física y mental y malas prospectivas económicas en la vida adulta (Trzesniewski et al., 2006).

Más allá de la relación que se pueda establecer entre las variables clínicas mencionadas y la autoestima, se ha considerado que el trabajo terapéutico en base de la autoestima genera excelentes resultados en diferentes grupos poblacionales. Así, por ejemplo, se ha trabajado en la promoción de la autoestima para tratar sintomatología depresiva (Hilbert, et al., 2019), en mujeres con anorexia nerviosa (Adamson et al., 2019) así como en menores en situación de riesgo social (González \& Gimeno, 2013). Estas investigaciones dan muestra de la fuerte necesidad de diagnosticar a temprana edad el nivel de autoestima y además de promover la autoestima como parte del desarrollo y bienestar integral de las personas.

El instrumento de evaluación más utilizado en el ámbito de promoción de la salud mental y en la práctica clínica es la escala de autoestima de Rosenberg (1965). Aunque la escala estaba inicialmente dirigida a adolescentes, su campo de aplicación se ha diversificado considerablemente, desde niños (Wu et al., 2016) hasta adultos y adultos mayores con o sin enfermedades (Classen et al., 2007; Gana et al., 2005; Magnusson \& Nermo, 2018; Wang et al., 2001) y personas con diferentes cuadros psicopatológicos (Ghaderi \& Scott, 2001; McCabe et al., 2007). La escala original de Rosenberg (1965) reportó la medida de un solo factor de autoestima medido a través de 10 ítems, 5 redactados de forma positiva y 5 de forma negativa. Sin embargo, existe evidencia que cuestiona la estructura factorial de la escala (Huang \& Dong, 2012). Así por ejemplo, investigadores en Colombia, señalaron que se trata de una escala de dos dimensiones: autoconfianza y autodesprecio (Ceballos, et al., 2017; Cogollo et al., 2015). Otros estudios encontraron evidencia de que existen 2 factores que subyacen a los ítems (Carmines \& Zeller, 1979; Hensley \& Roberts, 1976; Kaplan \& Pokorny, 1969; Salgado \& Iglesias, 1995).

Estudios diferentes señalan que se podría tratar de una escala unidimensional que cuenta con una estructura bifactorial (McKay et al., 2014), de manera que contienen factores positivos y negativos correlacionados en torno a un único constructo de autoestima. Diferentes autores consideran que estos hallazgos son en realidad resultado de errores en el diseño de investigación o efectos de método, debido, por ejemplo, a la presencia de preguntas formuladas en sentido positivo y en negativo (DiStefano \& Motl, 2006), lo que se observa sobretodo en minorías étnicas (Supple et al., 2013), o debido a la falta de habilidad lectora de los sujetos (Oliver et al., 
2012), o incluso, debido al procesamiento cognitivo de las personas evaluadas, puesto que las preguntas elaboradas en sentido negativo implicarían un cambio cognitivo imprevisto, ya que requieren mayor procesamiento mental $(\mathrm{Wu}$, 2008).

Esto tiene una gran signifiación puesto que implica que, una vez que se superan los efectos de método, la escala tendría una estructura unifactorial, como lo afirman gran cantidad de trabajos (Fleming \& Courtney, 1984; Hensley \& Roberts, 1976; Marsh, 1991; Shevlin et al., 1995; Tomas \& Oliver, 1999). En este sentido, Lima y Souza (2019), en un reciente estudio, señalaron que luego de aplicar la escala a más de 680 personas entre 18 y 70 años, determinaron que la escala posee una estructura unifactorial, toda vez que se controló el efecto de redacción (preguntas redactadas en positivo y negativo). Uno de los estudios más amplios para el análisis de la estructura factorial de la escala de autoestima de Rosenberg fue el planteado por Huang \& Dong (2012). Estos autores realizaon un meta-análisis de 23 estudios que daban cuenta de más de 32 mil participantes de 80 muestras independientes. Como resultados señalaron que la solución de un factor es la más recomendable. Otro interesante estudio llevado a cabo en población general y población clinica en Argentina estableció que existe un buen ajuste de los ítems a un modelo unidimensional, donde los items se agrupan en torno a un factor en cada grupo (Góngora \& Casullo, 2009). Finalmente, evidencia reciente propuso la evalación de 10 modelos distintos de estructura factorial de la escala de Rosenberg comparando los resultados con una serie de criterios externos estudiados en la literatura sobre autoestima. Los resultados mostraron que no existe evidencia de que el uso de diferentes estructuras internas tenga implicaciones sustanciales, lo que espera disminuir en parte las preocupaciones sobre la integridad de las conclusiones de la literatura de autoestima basada en los puntajes generales de la escala de Rosenberg (Donnellan et al., 2015).

Además de la estructura factorial, es importante considerar que para tener una medida detallada de la autoestima equivalente entre diferentes culturas, es necesario contar con una herramienta adecuada y adaptada al contexto en el que se investigue, ajustándose rigurosamente a directrices metodológicas, tal como señala la International Test Commission (2017). Así, se lograría que todos los resultados de estudios interculturales reflejen solo las diferencias o similitudes reales entre los grupos y no el producto de fallas de adaptación del instrumento (Eremenco et al., 2005). Por esta razón, durante las últimas décadas, la adaptación y validación de los instrumentos psicológicos sigue siendo de gran interés para los investigadores en el ámbito de la salud mental, aunque también resalta la preocupación por la calidad y la idoneidad de dichas adaptaciones (Borsa et al., 2012; International Test Commission, 2017). Por lo tanto, se propone el presente estudio con el propósito de evaluar las propiedades psicométricas de la Escala de Autoestima de Rosenberg (1965) en el contexto ecuatoriano, que incluya la evaluación confirmatoria de la estructura factorial, su validez de criterio, la evaluación de la invarianza entre las categorías de sexo y su consistencia interna.

\section{Método}

\section{Participantes}

La muestra está conformada por un total de 536 sujetos todos de nacionalidad ecuatoriana mayores de 18 años, con una edad promedio de 21.8 $(D E=4.7)$. Se trata de un muestreo no probabilístico por disponibilidad, las características fundamentales de la muestra se recogen en la Tabla 1.

Para garantizar un tamaño muestral suficientemente grande que permitiera efectuar los análisis estadísticos pertinentes a los objetivos planteados, así como generalizar hasta cierto punto las conclusiones obtenidas, se consideró contar con, al menos, 10 encuestados por cada ítem (Muñiz, 2005).

Tabla 1. Información de los sujetos participantes

\begin{tabular}{llcc}
\hline Variable & & $n$ & $\%$ \\
\hline \multirow{2}{*}{ Sexo } & Hombre & 130 & $24.4 \%$ \\
& Mujer & 405 & $75.6 \%$ \\
\hline \multirow{3}{*}{ Estado civil } & Soltero & 464 & $86.6 \%$ \\
& Casado & 43 & $8.0 \%$ \\
& Divorciado & 7 & $1.3 \%$ \\
& Unión libre & 21 & $3.9 \%$ \\
\hline
\end{tabular}




\section{Instrumentos}

Escala de Autoestima de Rosenberg (EAR): La escala original fue publicada por Rosenberg (1965) que incluye10 ítems, cinco redactados en sentido positivo y otros cinco en sentido negativo.

Se tomó en consideración la versión en español realizada por Atienza, Moreno y Balaguer (2000), esta versión al igual que la escala original consta de 10 ítems que se puntúan en escala tipo Likert del 1 al 4, además posee adecuadas propiedades psicométricas. Se decidió utilizar esta versión luego de la consulta a un panel de expertos quienes luego del respectivo análisis sugirieron utilizar esta traducción porque era la que mejor se ajustaba semánticamente a la cultura ecuatoriana.

Con esta versión se efectuó una prueba piloto y se evaluó la fiabilidad, validez y sensibilidad cultural. Además, se solicitó a cada participante información acerca de las dificultades que encontraban en responder a cada una de las preguntas. Este procedimiento se realizó considerando que la adaptación cultural es necesaria no sólo cuando se utilice el cuestionario en un idioma distinto, sino también en este caso por la diversidad cultural del español (Sousa \& Rojjanasrirat, 2011).

Otras escalas fueron consideradas para valorar a validez convergente, tales como:

Escala de Calidad de Vida: Se trata de la versión española del WHOQOLBREF (WHOQOL Group, 1996). La escala está formada por dos preguntas, una de ellas acerca de la calidad de vida global y la otra sobre la satisfacción con el estado de salud. Las 24 preguntas restantes corresponden a los cuatro dominios específicos: Físico, Psicológico, Social y Ambiente. Cada pregunta del WHOQOL-BREF posee una escala tipo Likert del uno a cinco (de "muy insatisfecho" a "muy satisfecho"; de "nada" a "completamente" y de "nada" a "extremadamente"). Se usó esta escala porque algunos Ítems de la dimensión de dominio Psicológico evalúan la autoestima.

Escala de apoyo social: Es la escala DUKEUNC, originalmente propuesta por Broadhead, Gehlbach, de Gruy y Kaplan (1988). Evalúa el Apoyo Social Confidencial, que es la posibilidad de contar con personas para comunicarse, y el Apoyo Social Afectivo, que son las demostraciones de amor, cariño y empatía. La escala de respuestas es tipo Likert con puntuaciones del 1 al 5. Para este estudio se usó la versión en español de Bellón, Delgado, Luna y Lardelli (1996).

Escala de Compromiso con la vida (LET): Se trata de una escala de 6 ítems diseñado para medir compromiso vital, entendido como el grado que la personas se compromete en actividades valiosas para sí mismos. Se responde en una escala Likert de 1 a 4 . Fue propuesto originalmente por Scheier, et al. (2006), y para este estudio se usará la versión en español de Lima-Castro, Vázquez y Blanco (2017).

\section{Procedimiento}

Para la aplicación de las pruebas se solicitó la autorización respectiva a las diferentes instituciones en las que se accedió a la muestra, como la Universidad de Cuenca, Universidad del Azuay, Hospital Vicente Corral Moscoso. Posteriormente se solicitó la firma de una carta de consentimiento informado a los sujetos en la cual se explicaban los fines investigativos de la evaluación y la confidencialidad de los datos obtenidos.

La aplicación se realizó de manera colectiva en salas de espera de consulta externa y salones de clases. Todos los instrumentos fueron autoadministrados. Cada participante los completó en 20 minutos aproximadamente.

El estudio se llevó a cabo en conformidad con la última revisión de la Declaración de Helsinki (AMM, 2017).

\section{Análisis de Datos}

Durante el tratamiento de las escalas se realizó una depuración de los valores perdidos, excluyendo aquellos casos en que los participantes dejaron de responder en al menos uno de los ítems de la escala, que concluyó con la eliminación de 14 observaciones de la muestra original.

En análisis de consistencia interna se calculan el coeficiente alfa de Cronbach $(\alpha)$ que se complementa con los coeficientes omega $(\omega)$ y omega jerárquico $\left(\omega_{\mathrm{h}}\right)$.

En la fase de evaluación de la bondad del ajuste del modelo se consideran los valores del error cuadrático medio de aproximación (RMSEA); el índice de ajuste comparativo (CFI), 
el índice Tucker-Lewis (TLI) y la raíz media cuadrática residual estandarizada (SRMR). Para efectos de interpretación se toma como referente los valores sugeridos por Hu y Bentler (1999) en los que el ajuste se considera que se obtiene un ajuste adecuado cuando el valor de RMSEA no excede de .06 , CFI y TLI son mayores a .95 y RMSR es inferior a .08 .

Para los modelos bifactor de la EAR, es decir, aquellos modelos que incluyen un factor general que afecta a todos los ítems y los modelos con factores que afectan solo a un grupo de ítems (Holzinger \& Swineford, 1937) se deben considerar estadísticos adicionales como la ECV (Explained Common Variance), PUC (Percentage of Uncontaminated Correlations), coeficiente $\mathrm{H}$ de replicabilidad de constructo, coeficiente omega $(\omega)$ y coeficiente omega jerárquico $\left(\omega_{\mathrm{h}}\right)$. En este sentido se esperan valores mayores a .7 para $\mathrm{H}$, ECV y PUC, estos dos últimos para respaldar la estructura esencialmente unidimensional de la EAR y valores mayores a .7 y .3 para omega y omega jerárquico que indican la varianza verdadera del modelo (Raykov \& Hancock, 2005; Rodriguez et al., 2016; Smits et al., 2015; Zinbarg et al., 2006)

La evaluación de la invarianza de la EAR se realizó en cuatro pasos: a) invarianza configural; b) invarianza métrica; c) invarianza escalar; y d) invarianza estricta. Valores de $\Delta \mathrm{CFI}<.01$ revelan ajuste absoluto y, por lo tanto, equivalencia entre grupos (Putnick \& Bornstein, 2016).

Se utilizó el método de mínimos cuadrados ponderados diagonalizados (DWLS) para la estimación debido a su precisión con variables ordinales y no-normales además de indicadores de bondad de ajuste más robustos para el tipo de datos mencionado (Mîndrilă, 2010). El análisis estadístico se realizó en el software R 3.6.0.

\section{Resultados}

\section{Prueba Piloto}

En la fase de estudio piloto la muestra estuvo constituida por 80 personas, $49 \%$ de población masculina $(n=39)$ y $51 \%$ de población femenina $(n=41)$ con edades comprendidas entre los 20 y 86 años, con una edad promedio de 43.88 $(D E=16.52)$. Esta muestra fue contactada en Hospital Público Vicente Corral Moscoso en el área de consulta externa, debido a la gran afluencia de personas que acuden a recibir atención y gracias al apoyo de las autoridades del hospital para realizar esta investigación.

La prueba piloto tuvo por finalidad realizar una evaluación preliminar de las características psicométricas de la escala evaluada, considerando fundamentalmente la confiablidad, la validez y la sensibilidad cultural y cada una de las acciones que implican estos conceptos. Cabe resaltar que ningún participante refirió dificultades en la compresión de cada uno de los ítems. El coeficiente alfa de Cronbach fue de .837. Para el cálculo del índice de discriminación se invirtieron los ítems negativos representados por los ítems 2, 5, 8, 9 y 10. Los índices de discriminación, usando la muestra completa, se muestran en la Tabla 2.

Tabla 2. Índices de Discriminación de los ítems de la Escala

\begin{tabular}{|c|c|c|c|}
\hline Ítem & $\begin{array}{c}\text { Dificultad del } \\
\text { ítem }\end{array}$ & Discriminación del ítem & $\begin{array}{l}\alpha \text { si el ítem es } \\
\text { eliminado }\end{array}$ \\
\hline P1. Me siento una persona tan valiosa como las otras & .86 & .70 & .81 \\
\hline $\begin{array}{l}\text { P2. Generalmente me inclino a pensar que soy un } \\
\text { fracaso }\end{array}$ & .83 & .62 & .81 \\
\hline P3. Creo que tengo algunas cualidades buenas & .85 & .52 & .83 \\
\hline P4. Soy capaz de hacer cosas tan bien como los demás & .86 & .66 & .81 \\
\hline $\begin{array}{l}\text { P5. Creo que no tengo mucho de lo que estar } \\
\text { orgulloso(a) }\end{array}$ & .77 & .45 & .83 \\
\hline P6. Tengo una actitud positiva hacia mí mismo(a) & .83 & .68 & .81 \\
\hline $\begin{array}{l}\text { P7. En general me siento satisfecho(a) conmigo } \\
\text { mismo(a) }\end{array}$ & .82 & 67 & .81 \\
\hline P8. Me gustaría tener más respeto por mí mismo(a) & .52 & .08 & .87 \\
\hline P9. Realmente me siento inútil en algunas ocasiones & .71 & .48 & .83 \\
\hline P10. A veces pienso que no sirvo para nada & .82 & .70 & .80 \\
\hline
\end{tabular}




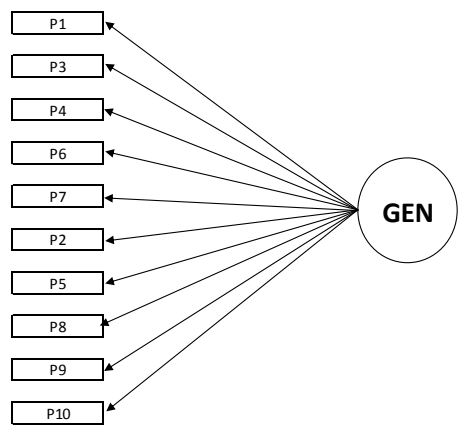

$1 \mathrm{a}$

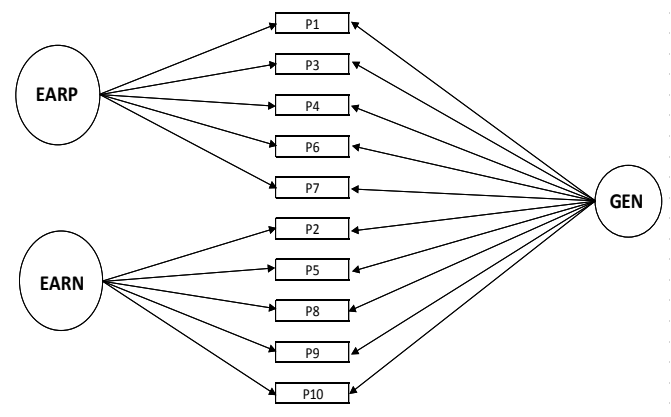

1c

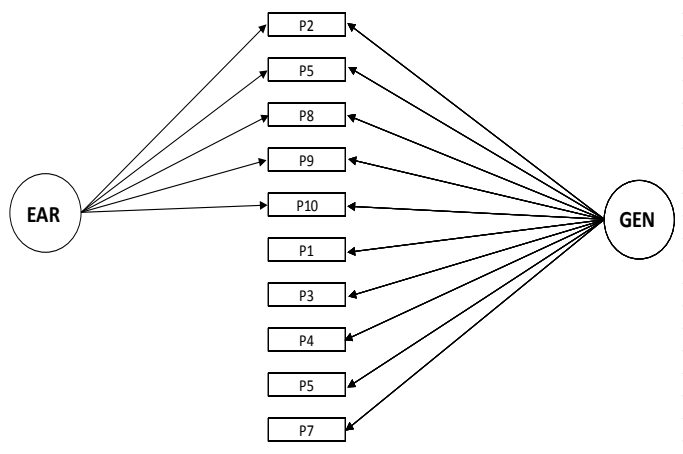

$1 \mathrm{e}$

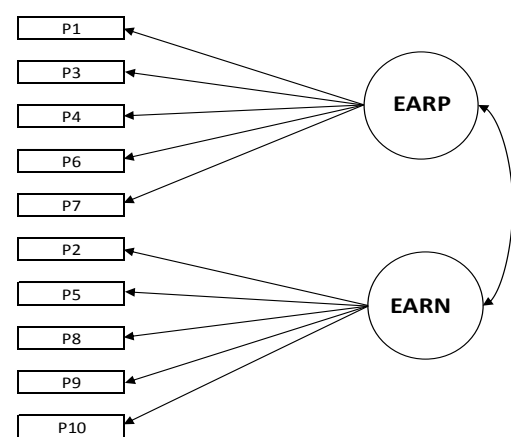

$1 b$

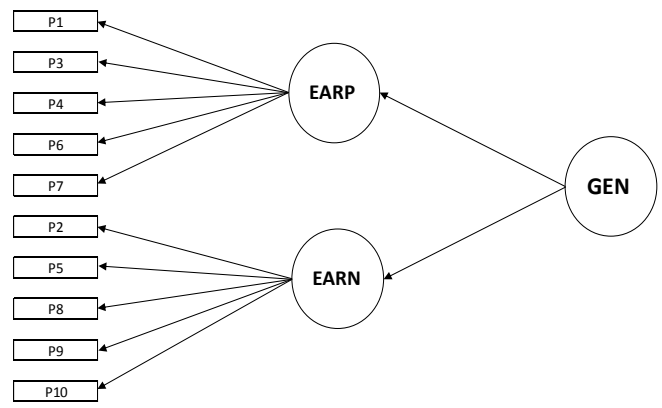

$1 d$

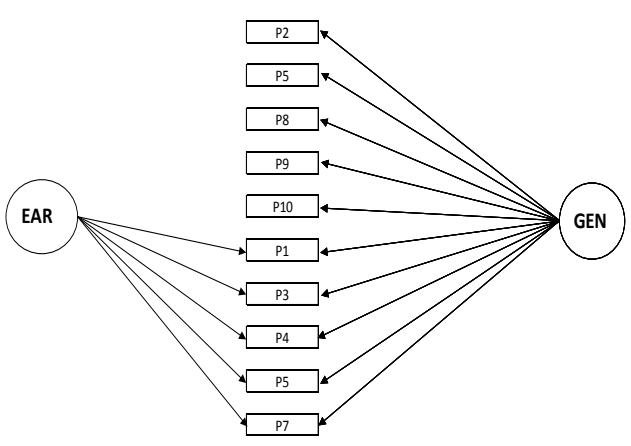

$1 f$

Figura 1. Modelos alternativos que fueron considerados en este estudio. El modelo 1 (1a) es el modelo original de un factor de primer orden que agrupa 10 ítems. El modelo 2 (1b) es un modelo de 2 factores de primer orden. El tercer modelo (1c) es un modelo bifactor. el modelo 4 (1d), es un modelo jerárquico con un factor de segundo orden. Finalmente, los modelos 5 y 6 (1e y $1 \mathrm{f}$ respectivamente) representan estructuras unifactoriales que controlan efectos de método con factores asociados a los ítems negativos y positivos.

\section{Análisis Factorial Confirmatorio}

La escala original ha sido sometida a múltiples estudios obteniendo soluciones distintas que se justifican según los contextos. Una evaluación de los modelos ajustados en la literatura fue considerada en este caso. Los modelos considerados se muestran en la Figura 1. El primer modelo (Figura 1a) muestra la estructura unifactorial original, el segundo modelo
(Figura 1b) refleja la estructura de dos factores de primer orden planteada por Carmines y Zeller (1979). La Figura 1c muestra el modelo bifactor (Hyland et al., 2014), el cuarto modelo considerado es un modelo jerárquico (Figura 1d). Finalmente, los modelos 5 y 6 evalúan una solución unifactorial con la inclusión de una variable latente para la corrección de efectos de 

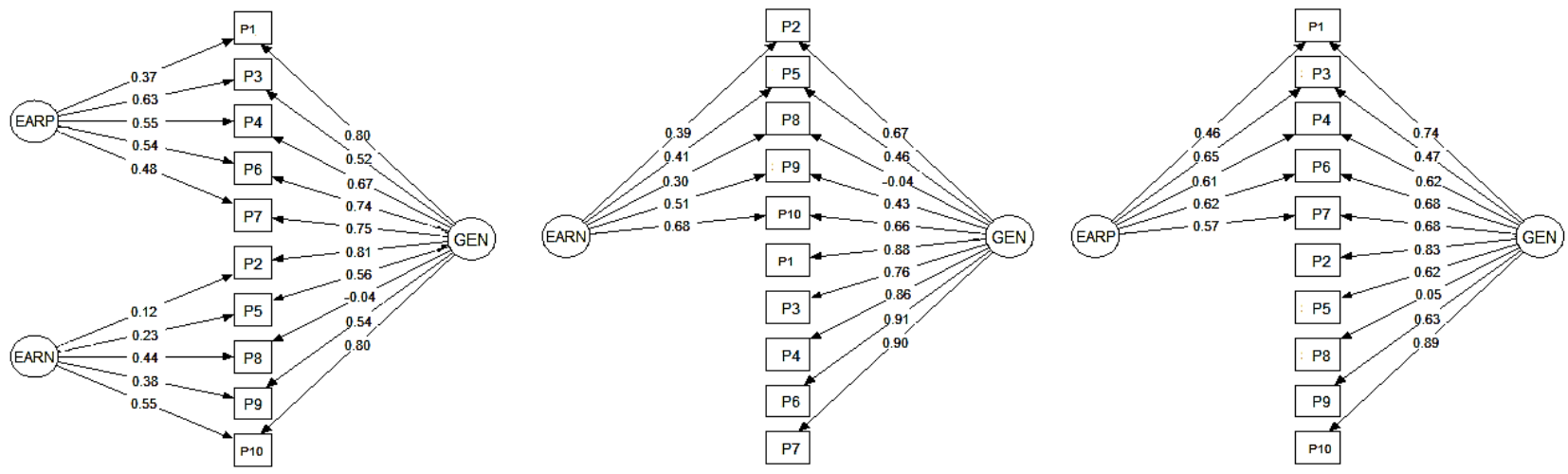

Figura 2. Modelo bifactor de la escala de autoestima de Rosenberg (Nota. Dimensión general de la Escala de Autoestima de Rosenberg. EARP: Variable latente para corrección de métodos de ítems positivos de la EAR. EARN: Variable latente para corrección de métodos de ítems negativos de la EAR)

Tabla 3. Índices de Bondad de ajuste para modelos de autoestima

\begin{tabular}{lccccccc}
\hline Modelo & $\chi^{2}$ & gl & $p$ & CFI & TLI & RMSEA & SRMR \\
\hline Dos factores & 116.44 & 34 & $<.01$ & .995 & .993 & .068 & .053 \\
Bifactor & 38.91 & 25 & .038 & .999 & .998 & .035 & .026 \\
Un factor (efecto de método ítems negativos) & 71.97 & 30 & $<.01$ & .997 & .996 & .052 & .037 \\
Un factor (efecto de método ítems positivos) & 83.56 & 30 & $<.01$ & .997 & .995 & .059 & .046 \\
\hline
\end{tabular}

Tabla 4. Índices de modelos bifactor

\begin{tabular}{lcccccc}
\hline Modelo & Factor & ECV & $\omega$ & $\omega \mathrm{h}$ & $\mathrm{H}$ & PUC \\
\hline \multirow{3}{*}{ Modelo 3 } & RSE - Positivo & .357 & .941 & & .664 & \\
& RSE - Negativo & .271 & .807 & & .475 & \\
& General & .678 & .930 & .741 & .908 & .556 \\
Modelo 5 & RSE - Negativo & .469 & .793 & & .628 & \\
& General & .816 & .924 & .822 & .950 & .778 \\
\multirow{2}{*}{ Modelo 6 } & RSE - Positivo & .453 & .940 & & .731 & \\
& General & .716 & .923 & .757 & .917 & .778 \\
\hline
\end{tabular}

Nota. ECV=Varianza extraída común; H=índice de replicabilidad de constructo; PUC=Porcentaje de correlaciones no contaminadas

método asociados a los ítems positivos y negativos (1e y 1f, respectivamente).

En el análisis confirmatorio de la solución unifactorial según el modelo original de Rosenberg (1965) observamos varios hallazgos interesantes; el ítem 8 con referencia al indicador "respeto consigo mismo" presenta una carga factorial baja (.103) en relación con los demás. Este modelo presenta la peor bondad de ajuste ya que el valor de RMSEA supera el valor máximo aceptable (RMSEA=.124). El modelo de un factor que excluye al ítem 8 y el modelo jerárquico también presentan valores de bondad de ajuste insuficiente (RMSEA=.135 y RMSEA =.128, respectivamente) por lo que deben descartarse.

Al evaluar la estructura del modelo bifactor, encontramos la mejor bondad de ajuste entre los modelos considerados (ver Tabla 3). En la evaluación adicional de los modelos bifactor se observa que ECV general y PUC supera el límite mínimo de 7 para los modelos con corrección de efectos de método; el índice $\mathrm{H}$ es mayor a .7 en los tres modelos evaluados; respecto a la consistencia interna de la EAR, los valores de los coeficientes omega y omega jerárquico superan los valores mínimos requeridos. Estos resultados proporcionan evidencia de que la EAR tiene una estructura factorial esencialmente unidimensional. El modelo bifactor que incluye una variable latente para la corrección de método en los ítems positivos presenta los mejores valores de bondad de ajuste (ver Tabla 4).

\section{Equivalencia}

La invarianza o equivalencia es la propiedad que permite determinar si el instrumento mantiene las propiedades psicométricas entre grupos. Los resultados se presentan en la Tabla 5 . 
Tabla 5. Evaluación de la invarianza de la escala entre las categorías de sexo

\begin{tabular}{lcccccc}
\hline & $\chi^{2}$ & $\mathrm{gl}$ & CFI & RMSEA & $\Delta$ CFI & $\Delta$ RMSEA \\
\hline Invarianza configural & 92.529 & 58 & .982 & .096 & NA & NA \\
Invarianza métrica & 101.861 & 71 & .982 & .081 & .002 & .015 \\
Invarianza escalar & 122.869 & 89 & .984 & .072 & $<0.01$ & .009 \\
Invarianza de medias latentes & 156.665 & 91 & .984 & .073 & .001 & 0 \\
\hline
\end{tabular}

Las restricciones impuestas a los modelos no indican un deterioro significativo $(\Delta \mathrm{CFI}<.01)$ en ninguno de los pasos, lo que evidencia invarianza de la EAR entre las categorías de sexo.

\section{Validez de criterio concurrente}

En la Tabla 6 se muestran las correlaciones entre la escala de EAR y tres escalas relacionadas con Calidad de Vida (WHOQOL), Propósito en la Vida (LET) y Apoyo Social (DUKE-UNC). La validez de criterio concurrente se determinó por las correlaciones significativas y positivas entre la dimensión positiva de la escala de autoestima de Rosenberg con los puntajes de las escalas LET, WHOQOL, y DUKE-UNC. Además, como era esperado, los ítems relacionados con el autodesprecio de la escala de autoestima de Rosenberg evidenciaron correlaciones significativas y negativas con dichas escalas.

Tabla 6. Correlaciones entre los ítems positivos y negativos de la EAR con Calidad de Vida, Apoyo Social y Propósito en la vida

\begin{tabular}{lccc}
\hline Factor/Criterio & WHOQOL & LET & DUKE \\
\hline Autoestima (EAR) & $.589 * *$ & $.709 * *$ & $.533^{* *}$ \\
\hline
\end{tabular}

** La correlación es significativa al nivel .01 (bilateral).

\section{Discusión y Conclusión}

El presente trabajo expone los resultados de la evaluación psicométrica de la escala de Autoestima de Rosenberg (1965) en el contexto ecuatoriano. Respecto a la consistencia interna, esta investigación ha demostrado que la escala presenta un valor alfa adecuado $(\alpha=.837)$, e incluso mayor a otras investigaciones de otros países en población general. Por ejemplo, en Chile presenta un alfa de 75 (Rojas-Barahona et al., 2009), mientras que en Argentina, se reportó un alfa de 7 (Góngora \& Casullo, 2009). También, el estudio comparativo de adaptación y validación de Colombia y España reportó un alfa de .86 y .83 (Gómez-Lugo, et al., 2016).
Además, se comprobó la validez de criterio concurrente con la solución unifactorial. Los resultados indicaron correlaciones moderadas y significativas, lo que va acorde a la teoría expuesta, puesto que la EAR correlaciona adecuadamente con los ítems de autoestima del WHOQOL-BREF. Así también, se evidenció una alta correlación entre la autoestima y el apoyo social percibido por la escala de DUKE-UNC, lo que se corrobora con investigaciones que aseguran que el apoyo social, brindado por los padres por ejemplo, es muy importante para el desarrollo de una buena autoestima en adolescentes (Valsala et al., 2018). Otros estudios apuntan a la fuerte relación que se establece entre el apoyo social junto a la autoestima como mediadores en la felicidad de las personas (Tan et al., 2017) y como factores protectores ante el estrés laboral ( $\mathrm{Su}$ et al., 2018). Finalmente, al investigar la correlación entre la EAR y la escala LET, se ha señalado que una baja autoestima generaría un impedimento para experimentar el sentido de vida, y por el contrario, altos niveles de autoestima influye de manera positiva en la felicidad, bienestar y en una mayor implicación laboral (Malonda \& Barahona, 2018).

La presente investigación incluye la evaluación de una estructura factorial de dos factores de primer orden: autoconfianza, y autodesprecio, según lo reportado en otras investigaciones (Alessandri et al., 2015; Carmines \& Zeller, 1979; Chao et al., 2017; Donnellan et al., 2015; Michaelides et al., 2016; RojasBarahona et al., 2009). Sin embargo, a pesar de que se presenta buena bondad de ajuste, los resultados de los modelos bifactor y de los modelos que incluyen una corrección de efectos de método son ligeramente superiores a las de otros estudios que encontraron evidencia de la estructura unifactorial original de la escala de Rosenberg en la que los ítems se configuraron en un sólo factor denominado autoestima global (Tomas \& Oliver, 1999). Esto fue respaldado por 
los valores obtenidos de ECV, índice H, PUC, omega y omega jerárquico.

En definitiva, la escala de autoestima de Rosenberg, es la medida de autoestima global más utilizada en la literatura, aunque aún persisten los desacuerdos sobre su estructura factorial interna (Donnellan et al., 2015).

Por otro lado, varios estudios señalan dificultades con el ítem 8 "respeto por sí mismo" (Ventura-León et al., 2018; Chao et al., 2017). En esta invesigación, este ítem presenta una carga baja en la solución bifactorial, además de presentar valores mediocres en los índices de dificultad y de discriminación. Por lo tanto, se sugiere utilizar una proposición más declarativa con menos adjetivación. Analizando el contenido del ítem, se observa que refleja una carga significativamente sesgada, representada por la frase "mí mismo", por lo que luego de consultar a expertos, familiarizados tanto con los términos y el constructo teórico del instrumento, y poseedores de conocimientos sobre las expresiones relacionadas con esos términos dentro del contexto ecuatoriano, se recomienda su cambio de redacción en la siguiente forma: "Me gustaría ser más respetuoso conmigo mismo", verificándose con este procedimiento, la equivalencia conceptual e idiomática de este ítem modificado, con el ítem de la escala original.

En cuanto a la invarianza de la escala, encontramos a medida que se incluyen restricciones, los cambios en el CFI son pequeños $(\triangle \mathrm{CFI}<0.01)$, lo cual revela invarianza configural, métrica, escalar y de medias latentes.

Finalmente, los resultados nos permiten concluir que la aplicación de la Escala de Autoestima de Rosenberg en el contexto ecuatoriano ofrece medidas fiables $y$ estructuralmente válidas. Por lo anterior, recomendamos este instrumento como adecuado para su uso en nuestro medio. Para efectos prácticos, se recomienda considerar un puntaje global de la EAR. En otras palabras, utilizar una estructura unifactorial.

En el futuro, otras investigaciones podrían extender el análisis de confiabilidad incluyendo pruebas test-retest $\mathrm{u}$ otras medidas de confiabilidad con datos obtenidos mediante muestreo aleatorio. Así mismo, se podría extender el análisis de equivalencia entre otros grupos demográficos (por ejemplo, por estratos sociales).

\section{Agradecimiento}

Los autores del trabajo agradecen a la Universidad del Azuay y a la Universidad de Cuenca por el apoyo y financiamiento recibido para el desarrollo de este estudio que formó parte del Proyecto de Investigación (Código DIUC_XV_2017 005) ganador del XV Concurso organizado por la Dirección de Investigación de la Universidad de Cuenca [DIUC].

\section{Referencias}

Adamson, J., Ozenc, C., Baillie, C., \& Tchanturia, K. (2019). Self-esteem group: Useful intervention for inpatients with anorexia nervosa? Brain Sciences, 9(1), E12. https://doi.org/10.3390/brainsci9010012

Alessandri, G., Vecchione, M., Eisenberg, N., \& Laguna, M. (2015). On the factor structure of the Rosenberg (1965) general self-esteem scale. Psychological Assessment, 27(2), 621635. https://doi.org/10.1037/pas0000073

Asociación Médica Mundial (AMM). (2017). Declaración de Helsinki de la AMM Principios éticos para las investigaciones médicas en seres humanos. febrero 06, 2020, de Asociación Médica Mundial Sitio web: https://www.wma.net/es/policiespost/declaracion-de-helsinki-de-la-ammprincipios-eticos-para-las-investigacionesmedicas-en-seres-humanos/

Atienza, F. L., Moreno, Y., \& Balaguer, I. (2000). Análisis de la dimensionalidad de la escala de autoestima de Rosenberg en una muestra de adolescentes valencianos. Revista de Psicología Universitas Tarraconensis, 22(12),

https://www.researchgate.net/publication/308 341043

Baumeister, R. F., Bratslavsky, E., Muraven, M., \& Tice, D. M. (1998). Ego depletion: Is the active self a limited resource? Journal of Personality and Social Psychology, 74(5), 1252-1265.

http://www.ncbi.nlm.nih.gov/pubmed/9599441 
Bellón, J., Delgado, A., Luna, J., \& Lardelli, P. (1996). Validez y fiabilidad del cuestionario de apoyo social funcional Duke-UNC-11. Atención Primaria, 18(4), 151-207. http://www.elsevier.es/es-revista-atencionprimaria-27-articulo-validez-fiabilidad-delcuestionario-apoyo-14325

Borsa, J., Damasio, B., \& Bandeira, D. (2012). Cross-cultural adaptation and validation of psychological instruments: Some considerations. Paidéia (Ribeirão Preto), 22(53), 423-432. https://doi.org/10.1590/198243272253201314

Brajsa-Zganec, A., Lipovcan, L., Ivanovic, D., \& Larsen, Z. (2017). Well-Being of nursing students: Role of affect regulation, selfesteem, family cohesion and social support. Open Public Health Journal, 10, 69-79. https://doi.org/10.2174/187494450171001006 9

Broadhead, W., Gehlbach, S., de Gruy, F., \& Kaplan, B. (1988). The Duke-UNC Functional Social Support Questionnaire. Measurement of social support in family medicine patients. Med Care, 26(7), 709-723. https://www.ncbi.nlm.nih.gov/pubmed/33930 31

Carmines, E., \& Zeller, R. (1979). Reliability and validity assessment. Sage.

Ceballos-Ospino, G., Barbosa, C., Suescún, J., Oviedo, H., Herazo, E., \& Campo-Arias, A. (2017). Validez y dimensionalidad de la escala de autoestima de Rosenberg en estudiantes universitarios. Pensamiento Psicológico, 15(2), 29-39. https://doi.org/10.11144/Javerianacali.PPSI15 -2.vdea

Chao, R., Vidacovich, C., \& Green, K. (2017). Rasch analysis of the rosenberg self-esteem scale with African Americans. Psychological Assessment, 29(3), 329-342. https://doi.org/10.1037/pas0000347

Classen, S., Velozo, C., \& Mann, W. (2007). The Rosenberg Self-Esteem Scale as a Measure of Self-Esteem for the Noninstitutionalized Elderly. Clinical Gerontologist, 31(1), 77-93. https://doi.org/10.1300/J018v31n01_06.

Cogollo, Z., Campo-Arias, A., \& Herazo, E. (2015). Escala de Rosenberg para autoestima: consistencia interna y dimensionalidad en estudiantes de Cartagena, Colombia. Psychologia: Avances de la Disciplina, 9(2), 61-71.

http://www.redalyc.org/articulo.oa?id=297241 658005

DiStefano, C. \& Motl, R. (2006). Further investigating method effects associated with megatively worded items on self-report surveys. Structural Equation Modeling: A Mutidisciplinary Journal, 13, 440-464. https://doi.org/10.1207/s15328007sem1303_6

Donnellan, B., Ackerman, R., \& Brecheen, C. (2015). Extending structural analyses of the Rosenberg Self-Esteem Scale to consider criterion-related validity: Can composite selfesteem scores be good enough? Journal of Personality Assessment, 98(2), 169-177. https://doi.org/10.1080/00223891.2015.10582 68

Drago, F. (2011). Self-esteem and earnings. Journal of Economic Psychology, 32(3), 480488. ttps://doi.org/10.1016/j.joep.2011.03.015

Eremenco, S., Cella, D., \& Arnold, B. (2005). A comprehensive method for the translation and cross-cultural validation of health status questionnaries. Evaluation \& the Health Professions, 28(2),

212-232. https://doi.org/10.1177/0163278705275342

Erol, R., \& Orth, U. (2016). Self-esteem and the quality of romantic relationships. European Psychologist, 21(4), 274-283. https://doi.org/10.1027/1016-9040/a000259.

Fleming, J., \& Courtney, B. (1984). The dimensionality of self-esteem: II. Hierarchical facet model for revised measurement scales. Journal of Personality and Social Psychology, 46(2), 404-421. https://doi.org/10.1037/0022-3514.46.2.404

Gana, K., Alaphilippe, D., \& Bailly, N. (2005). Factorial structure of the french version of the Rosenberg Self-Esteem Scale among the Elderly. International Journal of Testing, $5(2)$, 169-176. https://doi.org/10.1207/s15327574ijt0502_5.

Ghaderi, A., \& Scott, B. (2001). Prevalence, incidence and prospective risk factors for eating disorders. Acta Psychiatrica Scandinavica, $\quad 104, \quad 122-130$. 
https://www.ncbi.nlm.nih.gov/pubmed/11473 506

Gómez-Lugo, M., Espada, J., Morales, A., Marchal-Bertrand, L., Soler, F., \& VallejoMedina, P. (2016). Adaptation, validation, reliability and factorial equivalence of the Rosenberg Self-Esteem Scale in Colombian and Spanish population. The Spanish Journal of Psychology, 19(e66), 1-12. https://doi.org/10.1017/sjp.2016.67

Góngora, V., \& Casullo, M. (2009). Validación de la escala de autoestma de Rosenberg en población general y en población clínica de la ciudad de Buenos Aires. Revista Iberoamericana de Diagnóstico y Evaluación - e Avaliação Psicológica, 1(27), 179-194. http://www.redalyc.org/articulo.oa?id=459645 443010

González, F., \& Gimeno, A. (2013). Menores en situación de riesgo social: Valoración de un programa para la mejora de la autoestima. Psychosocial Intervention, 22, 1-5. https://doi.org/10.5093/in2013al

Hair, J., Black, W., Babin, B., Anderson, R., \& Tatham, R. (2006). Multivariate Data Analysis (6 ed.). Prentice Hall.

Hensley, W., \& Roberts, M. (1976). Dimensions of Rosenberg's Self-Esteem Scale. Psychological Reports, 38, 583-584. http://psych.ut.ee/ jyri/en/Paid2000_rses.pdf

Hilbert, S., Goerigk, S., Padberg, F., Nadjiri, A., Übleis, A., Jobst, A., Dewald-Kaufmann, J., Bühner, M., Naumann, F., Sarubin, N. (2019). The role of self-esteem in depression: A longitudinal study. Behavioural and Cognitive Psychotherapy, 47(2), 244-250. https://doi.org/10.1017/S1352465818000243

Holzinger, K. J., \& Swineford, F. (1937). The bifactor method. Psychometrika, 2(1), 41-54.

Hu, L., \& Bentler, P. (1999). Cutoff criteria for fit indexes in covariance structure analysis: Conventional criteria versus new alternatives. Structural Equation Modeling: A Multidisciplinary Journal, 6, 1-55. https://doi.org/10.1080/10705519909540118

Huang, C., \& Dong, N. (2012). Factor structures of the Rosenberg Self-Esteem Scale. European Journal of Psychological Assessment, $\quad 28(2), \quad$ 132-138. https://doi.org/10.1027/1015-5759/a000101
Hyland, P., Boduszek, D., Dhingra, K., Shevlin, M., \& Egan, A. (2014). A bifactor approach to modelling the Rosenberg Self Esteem Scale. Personality and Individual Differences, 66, 188-192.

https://doi.org/10.1016/J.PAID.2014.03.034

International Test Commission. (2017). The ITC Guidelines for Translating and Adapting [Segunda Edición]. Recuperado de International Test Commission. https://www.intestcom.org/files/guideline_test _adaptation_2ed.pdf

Kaplan, H., \& Pokorny, A. (1969). Selfderogation and psychosocial adjustment. Journal of Nervous and Mental Disease, 149(5), 421-434. https://doi.org/10.1097/00005053-19691100000006

Keane, L., \& Loades, M. (2017). Review: Low self-esteem and internalizing disorders in young people - a systematic review. Child and Adolescent Mental Health, 22, 4-15. https://doi.org/10.1111/camh.12204

Kenny, D. A., Kaniskan, B., \& McCoach, D. B. (2015). The performance of RMSEA in models with small degrees of freedom. Sociological Methods \& Research, 44(3), 486-507.

https://doi.org/10.1177/0049124114543236

Lima, T. J. S., \& Souza, L. E. C. (2019). Rosenberg Self-Esteem Scale: Method effect and gender invariance. Psico-USF, 24(3), 517-528. https://doi.org/10.1590/141382712019240309

Lima-Castro, S., Vázquez, F., \& Blanco, V. (2017). Adaptación, validación, confiabilidad y estructura factorial de la Escala de Compromiso Vital en población ecuatoriana. Manuscrito no publicado.

Lorenzo-Seva, U., \& Ferrando, P. (2006). Factor (Versión 7.0) [Software de computación]. Universitat Rovira i Virgili.

Magnusson, C., \& Nermo, M. (2018). From childhood to young adulthood: The importance of self-esteem during childhood for occupational achievements among young men and women. Journal of Youth Studies, 21(10), 1392-1410. https://doi.org/10.1080/13676261.2018.14688 76 
Malonda, Y., \& Barahona, N. (2018). Autoestima $\mathrm{y}$ sentido de la vida en estudiantes universitarios. Cauriensia, 13, 39-55. https://doi.org/10.17398/2340-4256.13.39

Marsh, H. (1991). Positive and negative selfesteem: A substantively meaningful distinction or Positive and negative selfesteem: A substantively meaningful distinction or artifactors? Journal of Personality and Social Psychology, 70, 810819.

https://www.ncbi.nlm.nih.gov/pubmed/86369 00

McCabe, R., Saidi, M., \& Priebe, S. (2007). Patients-reported outcomes in schizophrenia. British Journal of Psychiatry, 191(suppl. 50), s21-s28.

https://www.ncbi.nlm.nih.gov/pubmed/18019 040

McKay, M., Boduszek, D., \& Harvey, S. (2014). The Rosenberg Self-Esteem Scale: A bifactor answer to a two-factor question? Journal of Personality Assessment, 96(6), 654-660. https://doi.org/10.1080/00223891.2014.92343

Michaelides, M., Koutsogiorgi, C., \& Panayiotou, G. (2016). Method effects on an adaptation of the rosenberg self-esteem scale in Greek and the role of personality traits. Journal of Personality Assessment, 98(2), 178-188. https://doi.org/10.1080/00223891.2015.10892 48

Muñiz, J. (2005). Classical test models. In B. S. Everitt \& D. C. Howell (Eds.), Encyclopedia of Statistics in Behavioral Science (Vol. 1, pp. 278-282).

https://doi.org/10.1002/9781118445112.stat06 386.pub2

Mîndrilă, D. (2010). Maximum Likelihood (ML) and Diagonally Weighted Least Squares (DWLS) estimation procedures: A comparison of estimation bias with ordinal and multivariate non-normal data. International Journal for Digital Society, 1(1), 60-66. https://doi.org/10.20533/ijds.2040.2570.2010. 0010

Oh, K., Kim, Y., \& Kim, H. (2017). The effect of child care teachers' motivation for higher education and self-esteem on their happiness.
Advanced Science Letters, 23(3), 1690-1693. https://doi.org/10.1166/asl.2017.8629.

Oliver, A., Sancho, P., Galiana, L., Tomás, J., \& Gutiérrez, M. (2012). Efecto de método asociado a los ítems en negativo en la versión en portugués de la escala de autoestima de Rosenberg. Revista Iberoamericana de Diagnóstico y Evaluación - e Avaliação Psicológica (RIDEP), 1(33), 75-91. https://www.aidep.org/sites/default/files/201901/r33art4.pdf

Palacios, A., Pinedo, R., \& de la Iglesia, M. (2018). Exploring causes and consequences of sex workers' psychological health: Implications for health care policy. Health Care for Women International (22), 1-15. https://doi.org/10.1080/07399332.2018.14529 28

Pereira, A., Willhelm, A., Koller, S., \& Almeida, R. (2018). Risk and protective factors for suicide attempt in emerging adulthood. Ciencia e Saude Coletiva, 23(11), 3767-3777. https://doi.org/10.1590/1413-

812320182311.29112016

Putnick, D. L., \& Bornstein, M. H. (2016). Measurement invariance conventions and reporting: The state of the art and future directions for psychological research. Developmental review, 41, 71-90. https://doi.org/10.1016/j.dr.2016.06.004

Raykov, T., \& Hancock, G. R. (2005). Examining change in maximal reliability for multiplecomponent measuring instruments. British Journal of Mathematical and Statistical Psychology, 58(1), 65-82. https://doi.org/10.1348/000711005X38753

Robinson, J., Turner, J., Tian, Y., Neustadtl, A., Mun, S., \& Levine, B. (2019). The relationship between emotional and esteem social support messages and health. Health Communication, 34(2), 220-226. https://doi.org/10.1080/10410236.2017.14054 76

Rodriguez, A., Reise, S. P., \& Haviland, M. G. (2016). Evaluating bifactor models: Calculating and interpreting statistical indices. Psychological Methods, 21(2), 137-150. https://doi.org/10.1037/met0000045

Rojas-Barahona, C., Zegers, B., \& Förster, C. (2009). La escala de autoestima de 
Rosenberg: Validación para Chile en una muestra de jóvenes adultos, adultos y adultos mayores. Rev Med Chile, 137, 791-800. https://doi.org/10.4067/S003498872009000600009

Rosenberg, M. (1965). Society and the adolescent self-image. Princeton: Princeton University Press.

Rosenberg, M., Schooler, C., Schoenbach, C., \& Rosenberg, F. (1995). Global self-esteem and specific self-esteem: Different concepts, different outcomes. American Sociological Review, 60, 141-156. https://doi.org/10.2307/2096350

Salgado, J., \& Iglesias, M. (1995). Estructura factorial de la escala de autoestima Rosenberg: Un análisis factorial confirmatorio. Psicológica, 16, 441-454.

Scheier, M., Wrosch, C., Baum, A., Cohen, S., Martire, L., Matthews, K., . . Z Zdaniuk, B. (2006). The Life Engagement Test: Assesing purpose in life. Journal of Behavioral Medicine, 29(3), 291-298. https://doi.org/10.1007/s10865-005-9044-1

Shevlin, M., Bunting, B., \& Alan, C. (1995). Confirmatory factor analysis of the Rosenberg Self-Esteem Scale. Psychological Reports, 76, 707-710. https://doi.org/10.2466/pr0.1995.76.3.707

Smits, I. A. M., Timmerman, M. E., Barelds, D. P. H., \& Meijer, R. R. (2015). The Dutch symptom checklist-90-revised: Is the use of the subscales justified? European Journal of Psychological Assessment, 31(4), 263-271. https://doi.org/10.1027/1015-5759/a000233

Sousa, V., \& Rojjanasrirat, W. (2011). Translation, adaptation and validation of instruments or scales for use in cross-cultural health care research: A clear and user-friendly guideline. Journal of Evaluation in Clinical Practice, $\quad$ 17(2), 268-274. https://doi.org/10.1111/j.13652753.2010.01434.x

Sowislo, J., \& Orth, U. (2012). Does low selfesteem predict depression and anxiety? A meta-analysis of longitudinal studies. Psychological Bulletin, 139, 213-240. https://doi.org/10.1037/a0028931

Su, S., Feng, D., Wang, L., \& Liu, F. (2018). The protective role of self-esteem, perceived social support and job satisfaction against psychological distress among Chinese nurses. Journal of Nursing Management, 26(4), 366372. https://doi.org/10.1111/jonm. 12523

Supple, A. J., Su, J., Plunkett, S. W., Peterson, G. W., \& Bush, K. R. (2013) Factor structure of the Rosenberg Self-Esteem Scale. Journal of Cross-Cultural Psychology, 44(5), 748-764. https://doi.org/10.1177/0022022112468942

Tan, C., Krishnan, S., \& Lee, Q. (2017). The role of self-esteem and social support in the relationship between extraversion and happiness: A serial mediation model. Current Psychology, 36(3), 556-564. https://doi.org/10.1007/s12144-016-9444-0

Tomas, J., \& Oliver, A. (1999). Rosenberg's selfesteem scale: Two factors or method effects, structural equation modeling. $A$ Multidisciplinary Journal, 6(1), 84-98. https://doi.org/10.1080/10705519909540120

Tomas, J., Sancho, P., Galiana, L., Oliver, A., \& Hontangas, P. (2015). Measuring self-esteem in Spanish adolescents: Equivalence across gender and educational levels. Avaliação Psicológica, 14(3), 385-393. https://doi.org/10.15689/ap.2015.1403.10

Valsala, P., Devanathan, S., \& Kuttappan, S. (2018). Association of family challenges with self-esteem and perceived social support among Indian adolescentes. Child and Adolescente Social Work Journal, 35(6), 625$637 . \quad$ https://doi.org/10.1007/s10560-0180553-5

Ventura-León, J., Caycho-Rodríguez, T., Barboza-Palomino, M., \& Salas, G. (2018). Psychometric evidence of rosenberg selfesteem scale in adolescent from lima. Interamerican Journal of Psychology, 52, 4460. https://doi.org/10.30849/rip/ijp.v52i1.363

Waddell, G. (2006). Labor-market consequences of poor attitude and low self-esteem in youth. Economic Inquiry, 44, 69-97. https://doi.org/10.1093/ei/cbj005

Wang, J., Siegal, H., Falck, R., \& Carlson, R. (2001). Factorial structure of Rosenberg's Self-Esteem Scale among crack-cocaine drug users, structural equation modeling. $A$ Multidisciplinary Journal, 8(2), 275-286. https://doi.org/10.1207/S15328007SEM0802_ 6. 
WHOQOL Group. (1996). Versión Española del WHOQOL-Bref. Versión Barcelona. División de Salud Mental. Organización Mundial de la Salud. [Spanish language version of the WHOQOL-Bref. Mental Health Division. World Health Organization. Ginebra. https://www.who.int/mental_health/media/en/ 76.pdf

$\mathrm{Wu}, \mathrm{C} . \mathrm{H}$. (2008). An examination of the wording effect in the Rosenberg Self-Esteem Scale among culturally Chinese people. The Journal of Social Psychology, 148, 535-552. https://doi.org/10.3200/SOCP.148.5.535-552

Wu, Y., Zuo, B., Wen, F., \& Yan, L. (2016). Rosenberg Self-Esteem Scale: Method effects, factorial structure and scale invariance across migrant child and urban child populations in China. Journal of Personality Assessment, 111. https://doi.org/10.1080/00223891.2016.12174 20

Zinbarg, R. E., Yovel, I., Revelle, W., \& McDonald, R. P. (2006). Estimating generalizability to a latent variable common to all of a scale's indicators: A comparison of estimators for $\omega \mathrm{h}$. Applied Psychological Measurement, $\quad 30(2), \quad$ 121-144. https://doi.org/10.1177/0146621605278814 